\title{
Effects of Sample Storage on Spectral Reflectance Changes in Corn Leaves Excised From the Field
}

\author{
Matthew Lee ${ }^{1}$, Yanbo Huang ${ }^{1}$, Haibo Yao $^{2}$, Steven J. Thomson ${ }^{1} \&$ Lori M. Bruce ${ }^{2}$ \\ ${ }^{1}$ United States Department of Agriculture - Agricultural Research Service, Stoneville, Mississippi, USA \\ ${ }^{2}$ Geosystems Research Institute, Mississippi State University, Mississippi, USA \\ Correspondence: Yanbo Huang, United States Department of Agriculture - Agricultural Research Service, 141 \\ Experiment Station Rd., Stoneville, Mississippi, USA. E-mail: yanbo.huang@ars.usda.gov
}

Received: January 21, 2014 Accepted: June 11, 2014 Online Published: July 15, 2014

doi:10.5539/jas.v6n8p214 URL: http://dx.doi.org/10.5539/jas.v6n8p214

\begin{abstract}
Characterization of leaf spectra is useful for estimating spectra at the canopy level when viewed from airborne or space-borne sensors. When excising and transporting leaves to the laboratory, care must be taken so that degradation does not take place and alter the spectral signature. We compared the effect of elapsed time on leaf reflectance when excised corn leaves were stored inside and outside a cooler. Hyperspectral measurements were obtained 15 minutes after excision, then again 1, 2, 3, 4, 5, 6, and 24 hours after excision. Each hyperspectral band was modeled independently using a piecewise function with a linear portion for the first hour after plant excision and exponential portion after the first hour. Results showed that for the first hour, storage technique did not influence the signature. After the first hour, the leaves stored outside the cooler showed less change than leaves stored in the cooler. Furthermore, after approximately 30 minutes a large percentage of hyperspectral bands drifted beyond the level of significance (as determined by the mean plus or minus two standard deviations). These findings are valuable for developing methods for storage and analysis to support field studies and collection of ground-truth data to support remote sensing.
\end{abstract}

Keywords: hyperspectral signature, remote sensing, reflectance degradation

\section{Introduction}

Precision agriculture seeks to reduce costs by precisely controlling the agricultural inputs at subfield spatial resolution. This requires techniques that can efficiently determine the needs (water, fertilizers, and pesticides) of plants and precisely provide for them (Yao et al., 2012). Often, remotely sensing the needs of the plants is accomplished using optical sensors because of their ability to collect useful data on several plants within a relatively short period.

Hyperspectral sensors work by subdividing the optical portion of the electromagnetic spectrum, which includes ultraviolet, visible, and infrared, into many contiguous narrow bandwidth channels, or "bands." Since molecules and atoms absorb and reflect light at particular wavelengths based on their chemical formula, hyperspectral sensors can theoretically differentiate the structural and chemical composition of surfaces within their field of view. The capabilities of hyperspectral sensors degrade when they are used in remote sensing mode because of interference caused by the atmosphere, which the light interacts with as it traverses between the source (typically, the sun in remote sensing), object, and sensor (Gao et al., 2009). To minimize this interference, it may be useful to move the object to a laboratory setting, where the light source and sensor are closer to the object. In the case of plants, this typically involves excising samples such as leaves, stalks, branches, or stems from the plants because it is not feasible to transport intact plants to the laboratory.

Once a sample (such as a leaf or stalk) is excised from a plant, the sample experiences progressively increasing stress. The stress is present because the source of nutrition and moisture is cut off from the sample. Stress typically causes detectable changes in the reflectance spectrum of the samples, and these changes are often used to diagnose stress in crops caused by water, nitrogen, herbicides, or pesticides (Barnes et al., 2000). The presence of detectable stress in the samples could lead to incorrect conclusions about crops in the field. Thus, it is important to know how much time can be allowed to elapse after excision before the stress levels distort readings and thus prevent the samples from properly characterizing crops in the field. 
Previous studies have attempted to determine how much time should be allowed to elapse between excision of samples and measurement of their hyperspectral reflectance. However, most studies that the authors are aware of focused on common commercially grown plants. Thomasson and Sui (2009) modeled the spectrum of wilting cotton leaves using the SAS PROC MIXED procedure (SAS/STAT 9.2 User's Guide, Online), using a linear model. The authors rejected the hypothesis that the hyperspectral curve was significantly correlated with time. However if the spectral change is non-linear, it may be highly correlated with time. Lee et al. (2014) evaluated cotton and soybean leaves for two storage techniques: (1) storage of leaves inside paper bags placed inside a cooler with ice and (2) storage of the leaves inside paper, closable bags left outside a cooler. The study showed that leaves inside the cooler were preserved slightly better over long periods than the leaves outside the cooler. Over a short period, however, preservation was similar to leaves left outside the cooler. In both techniques, enough change to prevent the leaf samples from characterizing the crops was observed, so the long term advantage was not useful. Foley et al. (2006) preserved the leaves of common guava (Psidium guajava), purple guava (Psidium littorale), weeping fig (Ficus benjamina), floss silk (Chorisia speciosa), and coffee (Coffea arabica) by wrapping moist gauze around the petiole. The sample size used for each plant was one leaf for control and one leaf for treatment, but the study showed that response of leaves from different plant species varied significantly. The leaves also retained their general spectral curve when preserved with a moist paper towel wrapped around the petiole and placed in plastic bags better than when no preservation technique was used. Summy et al. (2011) examined many different storage combinations of giant reed (Arundo donax) involving different types of bags and refrigeration. The authors concluded that for most field campaigns where hyperspectral measurements are typically collected 24-48 hours after excision, storage within closable containers inside cooled ice chests is suitable for preserving leaf samples. However, the threshold of significant change was extremely wide at approximately four standard deviations. Such a threshold of significance may not be appropriate in difficult classification problems. Combined with the studies from Lee et al. (2014) and Foley et al. (2006), the research conducted by Summy et al. (2011) further reinforces the need to study spectral signatures of samples after they are excised, since every plant species has a different response. The objective of this research is to model the spectrum of corn leaves as a function of storage duration to provide guidance for field sample studies performed on corn crops.

\section{Experiment and Leaf Samples}

In the experiment, ten corn leaves were excised from plants in the farm of the USDA-ARS Crop Production Systems Research Unit located in Stoneville, MS, USA, and transported to a nearby lab. Five of the leaves were placed in paper bags and stored in a cooler at a measured temperature of 17.2 degrees Celsius. The cooler was kept cold using ice, with several layers of masking paper separating the leaf samples from the ice. The remaining five leaves were placed in similar paper bags not stored in a cooler. The ambient temperature outside the cooler was 22.9 degrees Celsius. The leaves were imaged using a hyperspectral camera (described herein) 15 minutes, 1 hour, 2 hours, 3 hours, 4 hours, 5 hours, 6 hours, and 24 hours after excision. After being imaged in the lab, the leaves were immediately placed back in their respective storage.

\section{Hyperspectral Imaging}

The leaves were imaged using a custom hyperspectral camera with an effective spectral range of 400-900 nm with 240 spectral bands. The camera is a 14-bit PCO1600 CCD (charge-coupled device) high resolution camera (Cooke Corporation, Romulus, MI, USA) that was integrated with an ImSpector V10E spectrograph (Spectral Imaging Ltd., Oulu, Finland) with a $30 \mu \mathrm{m}$ entrance slit and $23 \mathrm{~mm}$ Schneider lens. White and dark references were measured before data were collected so that reflectance images could be computed from images of the leaves. The white reference was measured using a Spectralon panel (Labsphere Inc., North Sutton, NH, USA), and the dark reference was measured by placing the lens cap on the camera. Reflectance $(R)$ was computed for each pixel using the formula below.

$$
R(\lambda)=\frac{D N_{P}(\lambda)-D C(\lambda)}{D N_{R}(\lambda)-D C(\lambda)}
$$

The central wavelength of the hyperspectral band is $\lambda$, and the digital numbers for the pixel and white reference are represented by $D N_{P}$ and $D N_{R}$, respectively, and the dark current is $D C$. The dark current and white references were collected once per day.

\section{Image Processing}

In order to isolate spectral curves of the corn leaves, the leaves were first segmented from the background. Segmentation was accomplished by thresholding Normalized Difference Vegetation Index (NDVI), a widely used parameter to represent plant vigor from remotely sensed data (Rouse et al., 1974). This produced a rough 
segmentation, which was cleaned up by visually comparing the segmentation to the image and manually correcting mislabeled pixels. The segmentation result was then used to identify pixels that were part of the leaf, which were then used to compute a mean spectral signature for the leaf. The spectral curves were then normalized by dividing by the value at $450 \mathrm{~nm}$ (Thomasson \& Sui, 2009). The mean spectral curves for the corn leaves stored in the cooler and outside the cooler are shown in Figure 1.

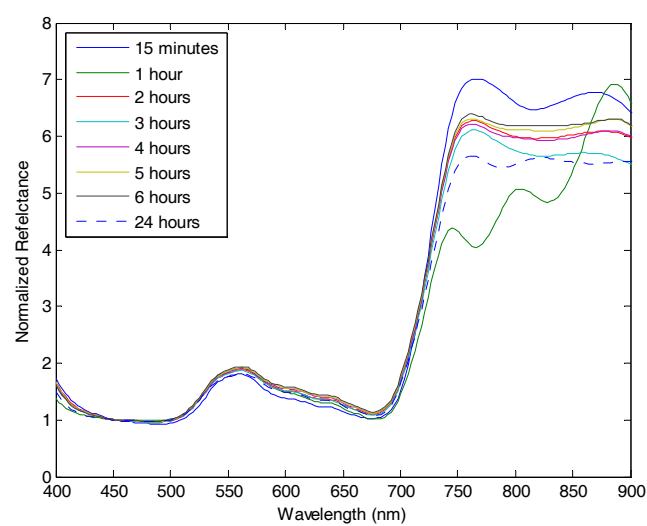

A.

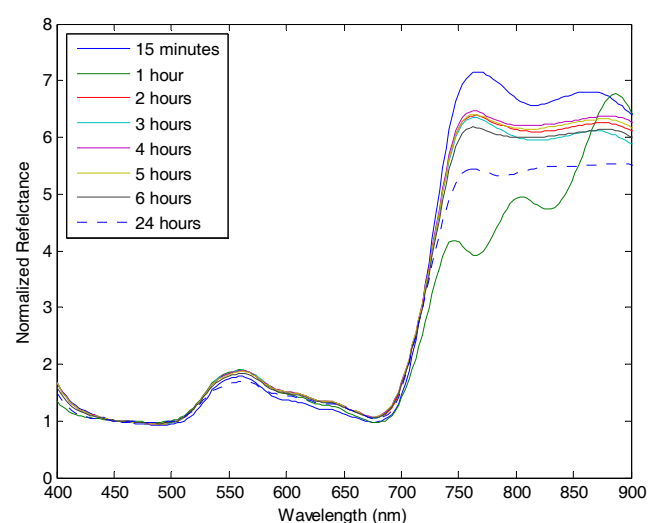

B.

Figure 1. Mean normalized reflectance signatures $(n=5)$ of corn leaves after various storage intervals: (A) stored inside a cooler (B) stored in a room outside the cooler. The signatures were normalized by dividing by the value at $450 \mathrm{~nm}$

\section{Data Modeling and Analysis}

The goal of the analysis was to estimate the time needed for each hyperspectral band to drift two standard deviations from its mean at first measurement (15 minutes after excision). When two classes have identical standard deviations and the means of both classes are separated by two standard deviations, the band will have an area under the receiver operating characteristic of 0.922 (Green \& Swets, 1966), a Bhattacharyya distance of 0.500 (Bhattacharyya, 1943), and result in classification accuracy of $84 \%$ using nearest mean classification (Duda et al., 2001) without any additional bands or features. Based on experience, classification problems with bands that have less separation are often difficult and require advanced classifiers (such as artificial neural networks and support vector machines) (Duda et al., 2001), while problems with bands of equal or better separation often produce very good classification results using simple techniques.

After normalized spectral signatures were obtained for each leaf at every time interval, analysis began by fitting the change in each hyperspectral band with respect to time to an appropriate model. Exponential models accurately described the change in each band for cotton and soybean leaves in a previous study (Lee et al., 2014). The corn leaves, however, consistently showed a spike in change occurring approximately one hour after excision, dissipating by two hours after excision (Figure 2). This spike prevented the change from fitting an exponential model well for most of the hyperspectral bands (as determined using T-test) (Montgomery \& Runger, 2003). However, when the data obtained one hour after excision are omitted, the remaining data does fit an exponential model. This situation led to the use of both a linear model (to model the change from 15 minutes to 1 hour) and an exponential model (to model the remaining time). 


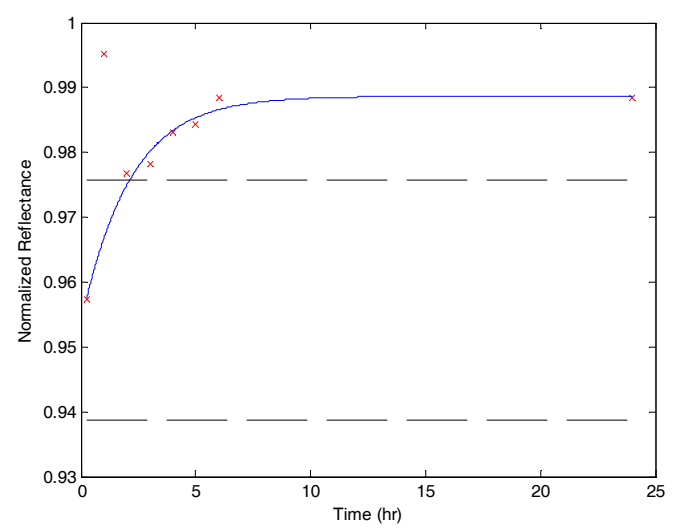

Figure 2. Plot of average reflectance with respect to time for hyperspectral band centered at $468.5 \mathrm{~nm}$. The average reflectance at $15 \mathrm{~min} ., 2 \mathrm{hr}$., $3 \mathrm{hr}$., $4 \mathrm{hr}$., $5 \mathrm{hr}$., $6 \mathrm{hr}$., and $24 \mathrm{hr}$. is a good fit to the exponential curve (blue line), but the average reflectance at $1 \mathrm{hr}$. does not fit well. The two standard deviation range is between the black dashed lines

The linear model was fit to the data points recorded 15 minutes and 1 hour after excision. Since only two measurement times were considered here, fitting the linear model only requires fitting a line through the means of both measurements. This is described by the equation

$$
N R(t)=\frac{N R_{1}-N R_{0.25}}{1-0.25}(t-0.25)+N R_{0.25}
$$

where $t$ is the time in hours, and $N R_{I}$ and $N R_{0.25}$ are the average normalized reflectance at 1 hour and 15 minutes ( 0.25 hours). Each normalized hyperspectral band $(N R)$ was modeled independently of other bands. In the analysis, we were only concerned with the amount of time needed to drift outside the plus or minus two standard deviation range. This means we did not need to model the "back end" of the spike. It would be safe to assume that the spike dissipates linearly or in any overall decreasing manner.

The exponential model is described by the equation,

$$
N R(t)=c_{1}+c_{2} e^{c_{3} t}
$$

Where $N R$ is the normalized reflectance for a single hyperspectral band, $t$ is the time in hours, and $c_{1}, c_{2}$, and $c_{3}$ are the model parameters. The parameters were chosen with non-linear least squares using data from 15 minutes, 2 hours, 3 hours, 4 hours, 5 hours, 6 hours, and 24 hours ( 1 hour was omitted). The accuracy of model parameters was evaluated using an F-test (Montgomery \& Runger, 2003).

The time required for the sample to drift outside the two standard deviation range was estimated by finding the intersection point between the model and the values determined by the 15 minute mean plus or minus two standard deviations. The critical time was determined by the amount of time elapsed between excision and the first time the model crossed this threshold. There are two equations in the model (the linear model for the interval from 15 minutes to 1 hour and the exponential model from 15 minutes to 24 hours without 1 hour). If the linear equation drifts outside the critical range within the first hour, the time the exponential equation drifts outside the normal range is insignificant since the linear equation will typically reach the threshold sooner. There is a possibility of bands deviating more than two standard deviations only to later return to the critical range. This scenario is not considered by the analysis since it is unlikely that all the bands will return to critical range at the same time. It is important to note that the model will only cross one critical value because the equations for the models are monotonic.

\section{Results and Discussion}

The data show that corn leaves cannot be described by an exponential model alone. This is apparent when examining the mean plots of the hyperspectral data for each time (Figure 1) because at 1 hour, the spectral signature is visually different in shape from any other time and because the T-test for the exponential model fails at 1 hour (Figure 3). Initially instrument error was the suspected cause, but this is not likely to have occurred because data collected after and before 1 hour after excision fits the exponential model also shown to model 
cotton and soybeans well (Lee et al., 2014). For the problem to be instrument error, the error would have to be present only for the 10 measurements at 1 hour, and no other time. This seems improbable since the anomaly was present in only part of the spectrum, laboratory conditions were consistent throughout the data collection process, and the same calibration was used at each imaging time. Furthermore, the F-test shows that the exponential model fits the data well if 1-hour data is removed (Figure 4).

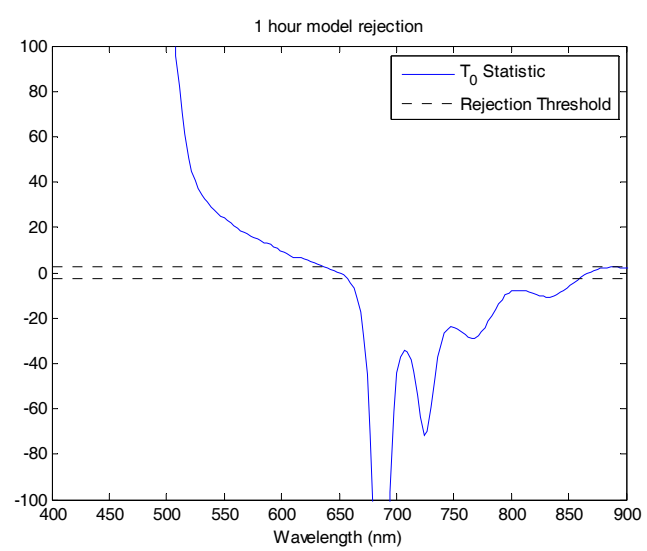

A.

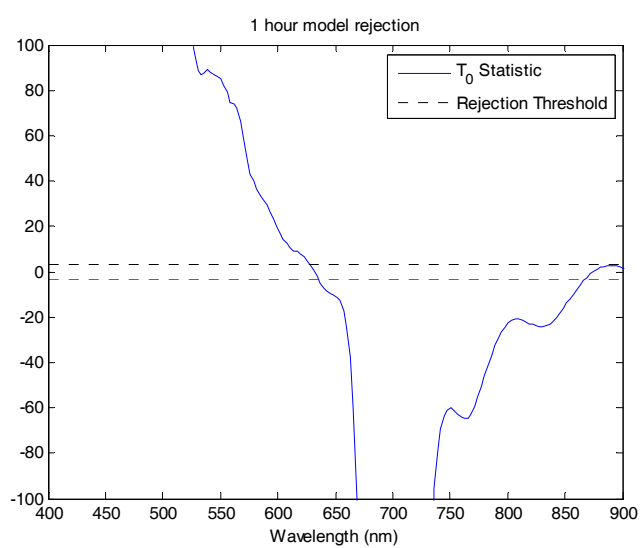

B.

Figure 3. T-test for exponential model using data collected at 1 hour after excision. The test uses a two sided 95\% confidence level. Corn leaves in A were stored inside a cooler, while the corn leaves in B were stored in a room outside the cooler

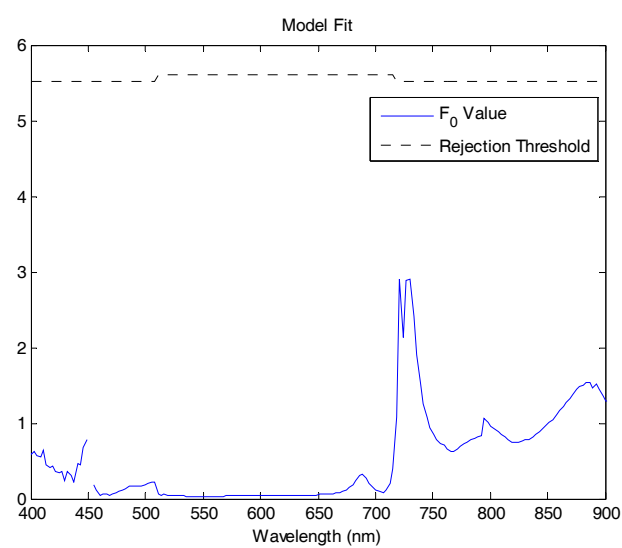

A.

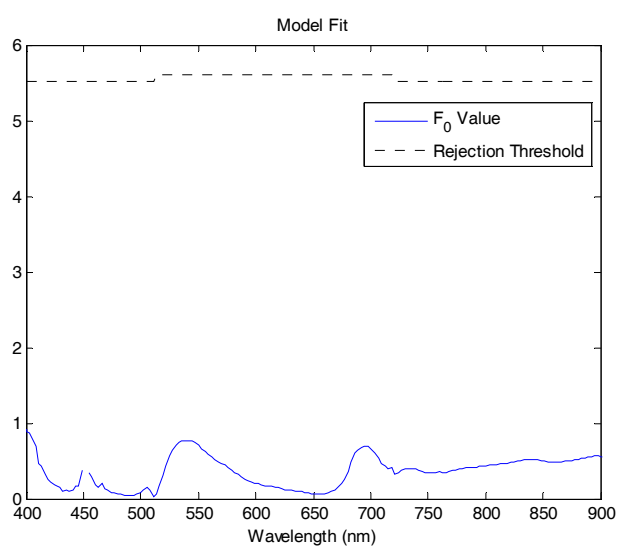

B.

Figure 4. F-test for exponential model using all of the corn data except that collected at 1 hour after excision. The confidence level for this test was $97.5 \%$. The leaves in A were stored inside a cooler, while the leaves in B were stored in a room outside the cooler

Out of a total of 175 bands, the number of bands eventually removed from the normal range between the time interval of 15 minutes to 24 hours was 148 for leaves stored in the cooler and 114 for leaves stored outside the cooler. Among the bands that drifted outside the normal range when stored in the cooler, 95 drifted outside the normal range within the first hour. For the leaves stored outside the cooler, 96 bands drifted outside the normal range within the first hour. Thus, both storage techniques performed virtually identically within the first hour. This observation is further emphasized by the plot of the drift time per band (Figure 5) and the cumulative results (Figure 6). Most of the hyperspectral bands drift outside their normal ranges more than 30 minutes after excision. After the first hour there is an advantage for leaves preserved outside the cooler. The majority of the difference is accounted for in the spectral range between 530-710 nm. This region of the spectrum is affected by photosynthesis in the plant, which may explain why placing leaves in a dark cooler affects reflectance more than leaving the leaves outside the cooler where there is light present. 


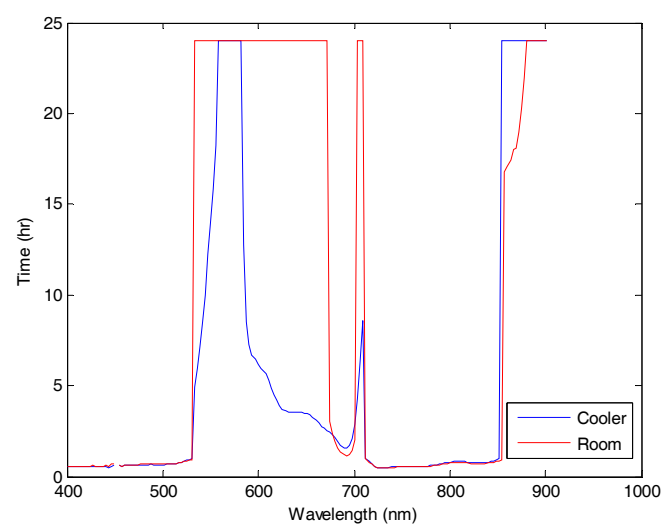

Figure 5. Plot of time required for spectral bands to drift outside the normal range for corn leaves. The blue and red lines represent corn leaves stored inside a cooler and in the room, outside the cooler, respectively

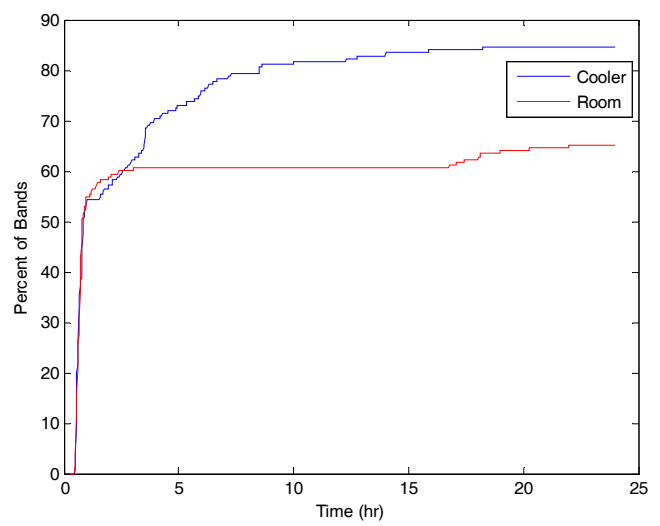

Figure 6. Estimated percent of bands outside the normal range with respect to time for corn leaves. The blue and red lines represent the corn leaves stored in a cooler and in the room, outside the cooler, respectively

\section{Conclusion}

This study shows that the exponential model used in previous research for soybean and cotton leaves does not fit the spectral decay of corn leaves well because the spectrum between 15 minutes and 1 hour after excision indicates a spike change that disappears 2 hours after excision. Corn leaves can thus be modeled using a combination of a linear model and exponential model. Results in Figure 5 reveal no advantage to storing corn leaves in a refrigerated cooler across the entire spectrum, and this implies that leaves should be processed within 30 minutes after excision. This does not mean that field collection campaigns must be limited to 30 minutes, but it simply means that steps must be taken to limit the time between excision and measurement for each leaf. Lab equipment might require close proximity to the field, and small quantities of leaves could be processed immediately. Figure 5 shows a window near both $600 \mathrm{~nm}$ and $900 \mathrm{~nm}$ where the spectrum of corn leaves is preserved very well. If these portions of the spectrum are the only portions of concern for a study, then the 30 minute limit does not apply.

Future work should entail repeating this experiment for a larger sample size and measuring the effects of storage duration on other agriculturally important plants. Effectiveness is limited by the spectral range of the camera system used (about $400-900 \mathrm{~nm}$ ). It may also be useful to study decay rate of the spectrum more closely to determine if (and how accurately) the original spectrum can be reconstructed based on time elapsed between excision and measurement within the measured spectrum.

\section{References}

Barnes, E. M., Clarke, T. R., Richards, S. E., Colaizzi, P. D., Haberland, J., Kostrzewski, M., ... Moran, M. S. (2000). Coincident detection of crop water stress, nitrogen status and canopy density using ground based 
multispectral data. Proceedings of the 5fh International Conference on Precision Agriculture. Retrieved from $\mathrm{http}: / / \mathrm{www} . c p r l . a r s . u s d a . g o v / p d f s / B a r n e s-c o i n c i d e n t \% 20$ detection.pdf

Bhattacharyya, A. (1943). On a measure of divergence between two statistical populations defined by probability distributions. Bulletin of Calcutta Mathematical Society, 35, 99-109.

Duda, R. O., Hart, P. E., \& Stork, D. G. (2001). Pattern Classification (2nd ed.). New York, NY: John Wiley \& Sons, Inc.

Foley, S., Rivard, B., Sanchez-Azofeifa, G. A., \& Calvo, J. (2006). Foliar spectral properties following leaf clipping and implications for handling techniques. Remote Sensing of Environment, 103, 265-275. http://dx.doi.org/10.1016/j.rse.2005.06.014

Gao, B., Montes, M. J., Davis, C. O., \& Goetz, A. F. H. (2009). Atmospheric correction algorithms for hyperspectral remote sensing data of land and ocean. Remote Sensing of Environment, 113, S17-S24. http://dx.doi.org/10.1016/j.rse.2007.12.015

Green, D. M., \& Swets, J. A. (1966). Signal Detection Theory and Psychophysics. New York, NY: John Wiley \& Sons, Inc.

Lee, M. A., Huang, Y., Yao, H., Thomson, S. J., \& Bruce, L. M. (2014). Determining optimal storage of cotton and soybean leaf samples for hyperspectral analysis. IEEE Journal of Selected Topics in Applied Earth Observations and Remote Sensing. Accepted for publication 29 May 2014.

Montgomery, D. C., \& Runger, G. C. (2003). Applied Statistics and Probability for Engineers (3rd ed.). New York, NY: John Wiley \& Sons, Inc.

Rouse, J. W., Haas, R. H., Schell, J. A., \& Deering, D. W. (1974). Monitoring vegetation systems in the Great Plains with ERTS. Proceedings of Third ERTS-1 Symposium, NASA SP-351, 309-317.

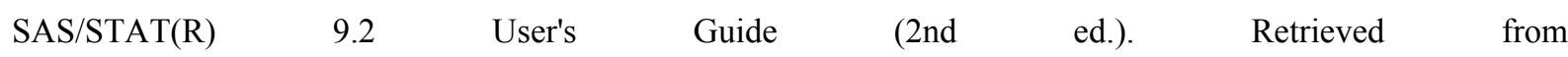
http://support.sas.com/documentation/cdl/en/statug/63033/HTML/default/viewer.htm\#mixed_toc.htm

Summy, K. R., Lieman, J., Gandy, Y. P., Mamachen, A., Mamachen, A., Goolsby, J., \& Moran, P. J. (2011). Effects of leaf excision and sample storage methods on spectral reflectance by foliage of giant reed, Arundo donax. Subtropical Plant Science, 63, 54-64.

Thomasson, J. A., \& Sui, R. (2009). Cotton leaf reflectance changes after removal from the plant. The Journal of Cotton Science, 13(3), 206-211.

Yao, H., Tang, L., Tian, L., Brown, R., Bhatnagar, D., \& Cleveland, T. (2012). Using hyperspectral data in precision farming applications. In P. S. Thenkabail, J. G. Lyon, \& A. Huete (Eds.), Hyperspectral Remote Sensing of Vegetation (pp. 591-607). Boca Raton, FL: CRE Press.

\section{Copyrights}

Copyright for this article is retained by the author(s), with first publication rights granted to the journal.

This is an open-access article distributed under the terms and conditions of the Creative Commons Attribution license (http://creativecommons.org/licenses/by/3.0/). 